\title{
CONCERNING CONTINUA IN A SEPARABLE SPACE WHICH DO NOT CROSS*
}

\author{
O. H. HAMILTON
}

In working with collections of continua it is sometimes useful to know something of the character of the point set consisting of all the points common to two or more members of the collection. Also it is of advantage to know conditions under which we may subtract a countable number of continua from the collection and have left a collection of mutually exclusive continua. The theorem which we shall prove may aid in answering questions of this nature.

All definitions and discussions will refer to point sets in a connected and locally connected separable space $S$.

DeFInITION 1. If $g_{1}$ and $g_{2}$ are two continua each of which separates $S$ ' $g_{1}$ will be said to cross $g_{2}$ provided there exist two complementary domains of $g_{2}$ (maximum connected domains of $S-g_{2}$ ) which contain points of $g_{1}$.

Definition 2. If (1) $M_{1}, M_{2}$, and $M_{3}$ are three point sets in $S$, (2) $g_{1}$ and $g_{2}$ are two continua in $S$, (3) $M_{1}$ is in a complementary domain $D_{1}$ of $g_{1}$ which does not contain a point of $g_{2}$, (4) $M_{2}$ is in a complementary domain $D_{2}$ of $g_{2}$ which does not contain a point of $g_{1}$, and (5) $M_{3}$ is in a complementary domain $D_{3}$ of $g_{1}+g_{2}$ distinct from $D_{1}$ or $D_{2}$, then $g_{1}+g_{2}$ will be said to separate $M_{1}, M_{2}$, and $M_{3}$ symmetrically with respect to $M$.

LEMMA 1. If (1) $g_{1}$ and $g_{2}$ are two continua each of which separates $S$, (2) each separates some complementary domain of the other, and (3) neither crosses the other, then there exist domains $D_{1}, D_{2}$, and $D_{3}$ such that $g_{1}+g_{2}$ separates $D_{1}, D_{2}$, and $D_{3}$ symmetrically with respect to $D_{3}$.

Proof. $S-g_{1}$ is the sum of two mutually separated point sets $S_{1}$ and $S_{2}$. One of these, say $S_{1}$, is such that the continuum $g_{2}$ is a subset of $S_{1}+g_{1}$. Let $D_{1}$ be a maximum connected domain of $S_{2}$. Also $S-g_{2}$ is the sum of two mutually separated point sets $S_{3}$ and $S_{4}$. One of these, say $S_{3}$, is such that $g_{1}$ is a subset of $S_{3}+g_{2}$. Let $D_{2}$ be a maximum connected domain of $S_{4}$. Let $D_{3}$ be a maximum connected domain of $g_{1}+g_{2}$ distinct from $D_{1}$ or $D_{2}$. We know $D_{3}$ exists, since by hypotheses each of the continua $g_{1}$ and $g_{2}$ separates some complementary domain of the other. The domains $D_{1}, D_{2}$, and $D_{3}$ are then separated by $g_{1}+g_{2}$ symmetrically with respect to $D_{\mathbf{3}}$.

* Presented to the Society, April 16, 1938. 
LEMma 2. If $g$ and h are two continua which do not cross and which have no complementary domain in common, and if $D$ is a complementary domain of $g$ such that h contains a point of $D$ and is a subset of $\bar{D}$, then $S-\bar{D}$ lies in a connected complementary domain of $h$.

Proof. Suppose $D_{1}$ and $D_{2}$ are two distinct complementary domains of $h$, each of which contains a point of $S-\bar{D}$. One of these domains does not contain a point of $g$, since $g$ and $h$ do not cross. Suppose $D_{2}$ contains no point of $g$. Then $D$ contains no point of $D_{2}$ and therefore contains no point of the boundary of $D_{2}$. Since, by hypothesis, every point of $h$ is in $D+g$, it follows that every point of the boundary of $D_{2}$ is in $g$ as well as in $h$, and therefore is in $g \cdot h$. Since we have supposed that $g$ contains no point of $D_{2}, h$ and $g$ have the complementary domain $D_{2}$ in common, and this contradicts a hypothesis of the lemma. We arrive at a similar contradiction if we suppose that $g$ contains no point of $D_{1}$.

Notation. In what follows, if $h_{1}$ and $h_{2}$ are any two continua whose sum separates the points $P_{1}, P_{2}$, and $P_{3}$ symmetrically with respect to $P_{3}$, then $D_{h 1}$ and $D_{h 2}$ will designate the complementary domains of $h_{1}+h_{2}$ which contain $P_{1}$ and $P_{2}$, respectively. A similar notation will be used if $h$ is replaced by any other symbol.

LEMma 3. If (1) $g_{1}, g_{2}, h_{1}$, and $h_{2}$ are four continua no one of which crosses another or has a complementary domain in common with another one of the four, (2) both $g_{1}+g_{2}$ and $h_{1}+h_{2}$ separate the points $P_{1}, P_{2}$, and $P_{3}$ symmetrically with respect to $P_{3}$, and (3) $h_{1} \cdot h_{2}$ contains a point not in $g_{1}+g_{2}$, then the domain $S_{1}=S-\left(h_{1}+h_{2}+D_{h 1}+D_{h_{2}}\right)$ is a proper subset of the domain $S_{2}=S-\left(g_{1}+g_{2}+D_{g 1}+D_{g 2}\right)$ and $h_{1}+h_{2}$ contains $g_{1} \cdot g_{2}$.

Proof. By hypothesis (3), $h_{1} \cdot h_{2}$ contains a point $P$ of some complementary domain of $g_{1}+g_{2}$. We know $P$ cannot be in $D_{g 1}$, for if so, then by Lemma $2, S-\bar{D}_{g 1}$, and therefore $P_{2}+P_{3}$, lies in a connected complementary domain $E_{2}$ of $h_{2}$. Since $h_{1}+h_{2}$ separates $P_{1}, P_{2}$, and $P_{3}$ symmetrically with respect to $P_{3}, \bar{E}_{2}$ does not contain $h_{1}$ (see Definition 2). Let $E_{1}$ designate the complementary domain of $h_{2}$ which with its boundary contains $h_{1}$. By Lemma $2, S-\bar{E}_{1}$, which contains $E_{2}$, lies in a connected complementary domain $E_{3}$ of $h_{1}$. That is, the complementary domain $E_{2}$ of $h_{2}$ which contains $P_{2}+P_{3}$ is a subset of the complementary domain $E_{3}$ of $h_{1}$. Then $h_{1}+h_{2}$ does not separate $P_{2}$ from $P_{3}$, and we have a contradiction of a hypothesis of the lemma. It follows that $h_{1} \cdot h_{2}$ cannot contain a point of $D_{g 1}$ and similarly cannot contain a point of $D_{g 2}$.

Then $h_{1} \cdot h_{2}$ must contain a point of $S_{2}$. Since $D_{g 1}$ and $D_{g 2}$ contain 
$P_{1}$ and $P_{2}$, respectively, and since $h_{1}$ separates $P_{1}$ from $P_{2}$ and contains no point of $D_{g 1}+D_{g 2}$, it follows that $D_{g 1}$ and $D_{g 2}$ lie in mutually exclusive complementary domains of $h_{1}$. There are points $Q_{1}$ and $Q_{2}$ on the boundaries of $D_{g 1}$ and $D_{o 2}$ which are in $g_{1}$ and $g_{2}$, respectively, but which are not points of $h_{1}$, since $h_{1}$ and $g_{1}$, and similarly $h_{1}$ and $g_{2}$, have no complementary domain in common. Then there exist mutually exclusive complementary domains $F_{1}$ and $F_{2}$ of $h_{1}$ such that $g_{1}$ and $g_{2}$ are subsets of $\bar{F}_{1}$ and $\bar{F}_{2}$, respectively. Consequently $g_{1} \cdot g_{2}$ is a subset of $\bar{F}_{1} \cdot \bar{F}_{2}$, that is, of $h_{1}$. Therefore, as required, $h_{1}+h_{2}$ contains $g_{1} \cdot g_{2}$. By hypothesis, $D_{h 1}$ and $D_{h 2}$ contain $P_{1}$ and $P_{2}$, respectively, and the sets $\bar{D}_{h 1}$ and $\bar{D}_{h 2}$ contain $D_{g 1}+g_{1}$ and $D_{g 2}+g_{2}$, respectively. Therefore $S_{1}$ is a subset of $S_{2}$; and $S_{1}$ is a proper subset of $S_{2}$, since by assumption $S_{2}$ contains a point of $h_{1} \cdot h_{2}$.

THEOREM. Let $G$ be a collection of continua having the following properties: (1) No continuum of $G$ crosses or contains a complementary domain in common with any other continuum of the coilection. (2) Each continuum of $G$ separates $S$ and also separates some complemenetary domain of each other continuum in the collection. Then there exists a countable collection $H$ of $G$ such that each point common to two continua of the collection $G$ is contained in some continuum of the collection $H$.*

Proof. Since $S$ is separable, there exists a countable subset $K$ of $S$ which has the property that every point of $S$ is a point of $K$ or a limit point of $K$. Let $L$ be a countable collection of the permutations of the points of $K$ taken three at a time. Let $p$ be any permutation $P_{1} P_{2} P_{3}$ of the collection $L$. Let $W_{p}$ be the collection of all sets each of which consists of two continua $a$ and $b$ of the collection $G$ such that $a \cdot b$ is not vacuous and such that $a+b$ separates $P_{1}, P_{2}$, and $P_{3}$ symmetrically with respect to $P_{3}$. In accordance with the notation used above, if $w_{\theta}=a_{\theta}+b_{\theta}$ is any set of the collection $W_{p}$, then $D_{\theta 1}$ and $D_{\theta 2}$ will designate the complementary domains of $a_{\theta}+b_{\theta}$ which contain $P_{1}$ and $P_{2}$, respectively, and $S_{\theta}$ will designate the domain $S-\left(D_{\theta 1}+D_{\theta 2}+a_{\theta}+b_{\theta}\right)$. A similar notation will be used if $w_{\theta}$ is replaced by any similar symbol. Let $\alpha$ be a well ordered sequence of the sets of the collection $W_{p}$. Let $\beta$ be a well ordered subsequence $v_{1}, v_{2}, \cdots, v_{\omega}, v_{\omega+1}, \cdots$ of $\alpha$. If $v_{\theta}=w_{\phi}$, let $c_{\theta}$ and $d_{\theta}$ designate $a_{\phi}$ and $b_{\phi}$, respectively. We shall define $v_{1}$ as being the first element of $\alpha$. Let $v_{2}$ be the first element of $\alpha$ which follows $v_{1}$ and has the property

* That the set of points each of which is common to two members of the collection $G$ is not necessarily countable may be seen if, in the cartesian plane, we define $G$ as being the collection of all continua, each of which is the sum of the $X$ axis between two points $A$ and $B$ and two vertical rays extending upward from $A$ and $B$. 
that $c_{2} \cdot d_{2}$ contains a point not in $c_{1}+d_{1}$. In general, if $\gamma$ is any ordinal number such that $v_{\psi}$ is defined for each ordinal number $\psi$ which precedes $\gamma$, let $v_{\gamma}$ be the first element of $\alpha$ which follows every $v_{\psi}$ and has the property that $c_{\gamma} \cdot d_{\gamma}$ contains a point not in any $c_{\psi}+d_{\psi}$. By Lemma 3 it follows that if $\theta$ and $\phi$ are any two ordinal numbers such that $\theta$ precedes $\phi$, then $c_{\phi}+d_{\phi}$ contains $c_{\theta} \cdot d_{\theta}$ and $S_{\phi}$ is a proper subset of $S_{\theta}$. Furthermore if $w_{\pi}$ is any element whatever of the sequence $\alpha$, then by virtue of the definition of the subsequence $\beta$, it follows that $a_{\pi} \cdot b_{\pi}$ is a subset of some set of the sequence $\beta$. By a well known property of abstract sets, since $S$ is separable, it follows that the sequence $\beta$ is countable. Let $H_{p}$ designate the countable collection of continua

$$
c_{1}, d_{1}, c_{2}, d_{2}, \cdots, c_{\omega}, d_{\omega}, c_{\omega+1}, d_{\omega+1}, \cdots .
$$

Let $H$ be the collection of all continua of the collection $G$ which are in $H_{p}$ for some permutation $p$ of the collection $L$. Then since $H_{p}$ is countable for each $p$ and since $L$ is countable, it follows that $H$ is countable.

Now let $h_{1}$ and $h_{2}$ be any two elements of the collection $G$ which have a point in common. Since $S$ is separable and locally connected, it follows from Lemma 1 that there exists a permutation $p, P_{1} P_{2} P_{3}$, of $L$ such that $h_{1}+h_{2}$ separates $P_{1}, P_{2}$, and $P_{3}$ symmetrically with respect to $P_{3}$. Consequently $h_{1}+h_{2}$ belongs to $W_{p}$ and $h_{1} \cdot h_{2}$ is a subset of some continuum of $G$ which belongs to $H_{p}$ and therefore is a subset of some continuum of $G$ which belongs to the countable collection $H$. The theorem is therefore true.

Corollaries. The following are obvious corollaries to the theorem:

COROLlaRY 1. If $G$ is any collection of continua satisfying the hypotheses of the theorem, then there exists a subcollection $G^{\prime}$ of mutually exclusive continua of $G$ which contains all but a countable number of the continua of $G$.

Corollary 2. If $G$ is a collection of continua in the euclidean plane, each of which is an open curve or a simple closed curve, and if no one of these continua crosses any other one of the collection, then there exists a countable subcollection $H$ of $G$ such that every point common to two elements of the collection $G$ is in some element of the collection $H$.

Examples. We see that the condition that the elements of $G$ do not cross is necessary if we let $G$ consist of all straight lines in the euclidean plane. It is to be noted that under these circumstances no two elements of $G$ have a complementary domain in common but 
each two nonparallel elements of $G$ cross each other. Obviously the conclusions of the theorem do not hold.

The following example will show that the condition that no two elements of the collection $G$ shall have a complementary domain in common is also necessary. In the cartesian plane let $M$ be a circle of radius 1 and center at the origin, and $N$ a circle of radius 1 and center at the point $(5,5)$. Let $G_{1}$ be a collection which contains each continuum which is the sum of $M$ and a horizontal straight line interval of length 10 whose left-hand end point is on the circle $M$ and which contains no point within $M$. Let $G_{2}$ be a collection which contains each continuum which is the sum of $N$ and a vertical straight line interval of length 10 whose upper end point is on the circle $N$ and which contains no point within $N$. Let $G=G_{1}+G_{2}$. No element of $G$ crosses any other element of $G$, but uncountably many have a complementary domain in common with some other element of the collection. However, it is evident that no countable subcollection of $G$ covers the set of points each of which is common to two continua of the collection $G$.

It is not known whether or not the condition that each element of $G$ shall separate some complementary domain of every other one can be omitted.

Oklahoma Agricultural and Mechanical College

\section{A PRINCIPAL AXIS TRANSFORMATION FOR NON-HERMITIAN MATRICES}

\section{CARL ECKART AND GALE YOUNG}

The availability of the principal axis transformation for hermitian matrices often simplifies the proof of theorems concerning them. In working with non-hermitian matrices (square or rectangular) it was found that a generalization of this transformation has a similar use for them.* A special case of this generalization has been investigated by Sylvester $\dagger$ who proved Theorem 1 (below) for square matrices with real elements. The unitary matrices $U$ and $V$ are in that case orthogonal matrices with real elements. Special cases had also been

* C. Eckart, The kinetic energy of polyatomic molecules, Physical Review, vol. 46 (1934), p. 383; C. Eckart and G. Young, The approximation of one matrix by another of lower rank, Psychometrika, vol. 1 (1936), p. 211; A. S. Householder and G. Young, Matrix approximation and latent roots, American Mathematical Monthly, vol. 45 (1938), p. 302.

† Sylvester, Messenger of Mathematics, vol. 19 (1889), p. 42. 